\title{
Advances in porous media science and engineering from InterPore2020 perspective
}

\author{
Jianchao Cai ${ }^{1,2 \oplus *}$, Hadi Hajibeygi ${ }^{3}$, Jun $\mathrm{Yao}^{4}$, S. Majid Hassanizadeh ${ }^{5}$ \\ ${ }^{1}$ State Key Laboratory of Petroleum Resources and Prospecting, China University of Petroleum, Beijing 102249, P. R. China \\ ${ }^{2}$ Institute of Geophysics and Geomatics, China University of Geosciences, Wuhan 430074, P. R. China \\ ${ }^{3}$ Faculty of Civil Engineering and Geosciences, Delft University of Technology, P. O. Box 5048, 2600 GA Delft, Netherlands \\ ${ }^{4}$ Research Center of Multiphase Flow in Porous Media, School of Petroleum Engineering, China University of Petroleum (East China), \\ Qingdao 266580, P. R. China \\ ${ }^{5}$ Department of Earth Sciences, Utrecht University, 3584 CD Utrecht, The Netherlands
}

Keywords:

Porous media

transport phenomena

multi-scale

modeling

Cited as:

Cai, J., Hajibeygi, H., Yao, J.,

Hassanizadeh, S.M. Advances in porous media science and engineering from InterPore2020 perspective. Advances in Geo-Energy Research, 2020, 4(4): 352-355, doi: 10.46690/ager.2020.04.02.

\begin{abstract}
:
Natural, artificial, and biological porous media can be seen everywhere in our daily lives. Transport phenomena in porous media, such as flow, diffusion, reaction, adsorption and deformation, are encountered in a wide variety of practical applications and scientific interests over widely disparate length scales, from molecular, to pore, core, and field scales. However, determination of transport properties in porous media remains a challenging issue. During the $12^{\text {th }}$ Annual Meeting of the International Society for Porous Media (InterPore), held online from August 31-September 4, 2020, advances on porous media science and engineering in very broad areas were presented. The meeting was attended by more than 750 participants from across the globe, and a significant milestone was achieved in the history of InterPore conferences due to its online interactive platform. Participants could access the pre-recorded talks, leave comments and questions, chat with each other, one week before the conference. Then, all the feedback related to a talk was discussed in the presence of the author during several Q\&A sessions. Invited and Keynote talks were live, and were also recorded. Each Q\&A session was moderated by two experts, who first reviewed the 8 contributions of their session and then summarized the questions for each talk. The author could further elaborate their work and answer the questions.
\end{abstract}

\section{Introduction}

Porous media are solid materials composed of interconnected pores which are typically filled with fluids. Porous media can be found in many natural and industrial systems, such as subsurface reservoirs, artificial and engineering materials, biological tissues and plants (Ingham and Pop, 1998; Vafai, 2015). The pioneering studies on porous media can go back to the 19th century (Darcy, 1856). Due to the extremely complex pore structures and fluid distribution in many porous systems and their wide range of applications, major breakthroughs in theories and models are yet needed (Bear and Bachmat, 2012). These breakthroughs need to involve new concepts, modeling approaches, and experimental techniques, accounting for multi-scale and multi-disciplinary nature of the modern challenges with which our society is facing (Das and Hassanizadeh, 2005; Vadász, 2008).

Under this background, the non-profit-making scientific organization, the International Society for Porous Media (InterPore), was established in 2008, in order to advance and disseminate knowledge for understanding, descripting, and modeling natural and industrial porous systems. The InterPore organization presents a platform for researchers active in modeling flow and transport in various porous media, in which the annual meeting is one of the most important activities since 2009.

During August 31 -September 4, 2020, the $12^{\text {th }}$ Annual Meeting (hereafter InterPore2020) was held online. Several coherent complementary minisymposia, invited lectures, keynote

\begin{tabular}{c|} 
Yandy \\
Scientific \\
Press
\end{tabular}

${ }^{*}$ Corresponding author.

E-mail address: caijc2016@hotmail.com (J. Cai); H.Hajibeygi@tudelft.nl (H. Hajibeygi); rcogfrupc@126.com (J. Yao);

S.M.Hassanizadeh@uu.nl (S.M. Hassanizadeh).

2207-9963 (C) The Author(s) 2020.

Received September 10, 2020; revised September 11, 2020; accepted September 11, 2020; available online September 13, 2020. 
lectures, invited poster pitch presentations, award ceremony, exhibitions, writing workshop, career development event, and Q\&A sessions are the main components of this meeting.

\section{Minisymposia: extensive themes on porous media}

The minisymposia, each based on a theme and topic on porous media, are extensive, ranging from flow, transport and thermal properties, to multi-scale, multi-physics, nonlinear and coupled processes. Further, biochemical and reaction processes, interfacial phenomena, modeling and imaging techniques, and utilization of porous media for energy, climatedriven research, ecohydrology and biogeochemistry of agroecosystems are also among them. The list of all minisymposia follows:

- Porous media for a green world: energy \& climate

- Porous media for a green world: water \& agriculture

- Flow, transport and mechanics in fractured porous media

- Swelling and shrinking porous media

- Biochemical processes and biofilms in porous media

- Physics of multi-phase flow in diverse porous media

- Interfacial phenomena in multiphase systems

- Mathematical and numerical methods for multi-scale multi-physics, nonlinear coupled processes

- Mixing, dispersion and reaction processes across scales in heterogeneous and fractured media

- Pore-scale modelling

- Advances in imaging porous media: techniques, software and case studies

- Microfluidics in porous systems

- Advances in modeling and simulation of poromechanics

- Fluids in nano-porous media

- Uncertainty quantification in porous media

- Machine learning and big data in porous media

- Fluid interactions with thin porous media

- Thermal processes, thermal coupling and thermal properties of porous media: modeling and experiments at different scales

- Innovative methods for characterization, monitoring, and in-situ remediation of contaminated soils and aquifers

- Electrochemical processes in porous media

- Biophysics of living porous media: theory, experiment, modeling and characterization

- Effective elastic, thermal, electrical and optical properties of porous materials, cellular materials, foams and metamaterials

- Catalysis and adsorption/absorption processes in porous media

Besides the topics on porous media, a special session was organized in recognition of professor Rainer Helmig's contributions in porous media science.

3. Invited lecture: from basic porous flow in different scales to emerging technologies for global water problem

In this section, ten researchers introduced the current advances on a variety of flow and transport processes in porous media on different scales and perspectives.

Moran Wang, from Tsinghua University, presented a talk entitled "Electrokinetic and ion transport in micro/nanoporous media". He discussed electrokinetic and interface theories for ion transport in a mesoscopic numerical framework for predictions. Validations by comparing with theories and experimental data, and multiscale analysis in both spatial and temporal scales for special applications are presented.

About the wettability, contact line dynamics and surface tension during the pore-scale motion of interfaces, Stéphane Zaleski, from Sorbonne University, reported the advances of his group on "Contact line motion using the Volume of Fluid method". A slip length model was discussed in various regimes and a sharp interface model was implemented using a volume of fluid method in the context of several open-source codes. Despite significant advancements, in the discussion section, Stéphane highlighted that further research is still needed to consistently connect the multiphase pore-scale system to continuum scale and to cover a wider range of physically challenging transport phenomenon.

In a lecture entitled "Multiscale in-situ fluid monitoring to understand and model multiphase flow in porous media", Olga Vizika, from IFP Energies Nouvelles, combined experimental observation and theoretical analysis, with selected examples at different scales, and demonstrated the necessity of adapting space and time scales to study multiphase flow in porous media, and discussed the complementarity of different methods.

In the lecture of "Porous media, small and large: From atomistic modeling of nano-porous membranes to modeling of flow and transport in geological formations" by Muhammad Sahimi, from University of Southern California, four classes of fundamental problems of flow, transport, reaction, adsorption and deformation in porous media were described and the corresponding modeling approaches were discussed. The challenge of upscaling from core to field scale was also discussed. It should be noted that Mohammad is the recipient of the Kimberley-Clark distinguished lectureship award and will give invited lectures in 2021 (see interpore.org/interporefoundation).

In "Topology and its effects on fluid flow", James McClure, from Virginia Polytechnic Institute and State University, presented the topological changes in multiphase flow in porous media in the context of geometric evolution, and used geometric laws to derive the relationship between geometric invariants. Noting that changes to the topology of an object occur as discrete events, the fundamental mechanisms that drive these changes need to be considered in detail. The associated physical consequences were examined from the molecular scale, the pore-scale, and the reservoir scale.

In "Coupling free flow and porous-media flow, and its applications to aerospace and mechanical engineering", Guang Yang, Shanghai Jiao Tong University, presented fundamental research on the momentum transfer characteristics at the coupling interface, the roles of this coupling effect in three specific applications, and the progress of parameter optimization studies.

For the "Facilitating the Reproduction of Simulation Re- 
sults", Bernd Flemisch, from University of Stuttgart, presented the measurement results obtained with the open-source porousmedia simulator Dumux. The current status on the study of flow and transport in fractured porous media was also illustrated.

In "A posteriori error estimates and adaptive solvers for porous media flows", Martin Vohralík, from Inria Paris, presented several porous media flow systems, including the steady linear single-phase Darcy flow, passing through problems involving reaction, advection, nonlinearities, degeneracies, or thermal effects, and multi-phase multi-compositional flows with phase change. He also presented a unified framework covering common discretization schemes, discussed general polygonal/polyhedral meshes, and addressed cheap implementations of the estimates.

Fred Vermolen, from University of Hasselt and Delft University of Technology, delivered a lecture on "Modelling the behaviour of skin after deep tissue injury using poroand morpho-elastic models" and presented a morpho-elastic model and the underlying mathematical and numerical issues to simulate human skin mechanics and healing processes.

Reporting on "HydroGrid: Emerging technologies for global water quality and sustainability", Lilit Yeghiazarian, from University of Cincinnati, introduced the concept of HydroGrid as a framework to describe connected water systems, outlined mirroring concepts in the HydroGrid. She also discussed emerging technologies in advanced materials, porous media design and modeling, signal transduction and wireless communications.

\section{Keynote lecture: from flow simulation and material design to water scarcity problem and organ transport models}

Pore-scale direct numerical simulation is a potentially powerful numerical tool to analyze flow and transport processes in various energy and environmental systems. In "Pore-scale direct numerical simulation of flow and transport in energy and environment", Qinjun Kang, from Los Alamos National Laboratory, presented a state-of-the-art research on pore-scale direct numerical simulation of various flow and transport phenomena. This included supercritical $\mathrm{CO}_{2}$ displacing brine in a heterogeneous micromodel and real rock, oil and water two-phase flow in fractionally wet porous media, corrosion of uranium dioxide in a geological repository, multiphase multicomponent reactive transport during $\mathrm{CO}_{2}$ dissolution trapping, as well as hydrocarbon behavior in nanopores of tight reservoirs.

Heterogeneous properties of polymer electrolyte membrane fuel cell and electrolyzers and their interfacial contacts have great influence on electrochemical performance. Aimy Bazylak, from University of Toronto, gave the presentation on "Designing porous materials for improved fuel cell and electrolyzer performance" to discuss how the heterogeneous porous materials and nature of interfacial contacts influence the flow and mass transport behavior in polymer electrolyte membrane fuel cells and electrolyzers, and introduced their new designed and fabricated materials.
Besides the general pressure difference, chemical potential difference, electric and gravitational fields for flow and transport in porous media, thermal driving forces are also widely existing and encountered. In "Addressing the water scarcity problem with thermal osmosis", Signe Kjelstrup, from Norwegian University of Science and Technology, focused on the thermal osmosis, examined the theoretical and practical conditions for a particularly important case of two-phase flow in porous media, the flow of water using vapor-gap membranes and a waste heat source to provide a driving force. She argued that the theoretical basis of non-equilibrium thermodynamics can help understand the mechanism of thermal osmosis.

Reliable and accurate diagnosis of diseases is important for the early detection and targeted treatment of diseases. In "Microscopical lesions of the transport system of organs and their relation to clinically observable large-scale phenomena", Dominik Obrist, from University of Bern, presented multiscale models to infer the state of microscopical lesions from observable large-scale phenomena, and presented several computational and experimental multi-scale models of organs. He demonstrated how they can be used to support the diagnosis of diseases of the brain, heart and lung.

\section{Invited poster presentation pitch: Flow diagnostics and methodology for flow in porous media in different scales}

Due to the complex and uncertain geology factors of fractured reservoirs, it is very difficult to characterize, develop, and manage them. In the "Flow diagnostics for fractured reservoirs: An innovative way to account for geological and geo-mechanical uncertainty in modern reservoir modelling and simulation workflows", Sebastian Geiger, from HeriotWatt University, introduced a new flow diagnostics tool for naturally fractured reservoirs which computed some of the essential dynamic reservoir behaviours. His new flow diagnostics framework also accounts for geomechanical effects in the reservoir. This new technology could screen large numbers of geological models based on their approximate dynamic and geomechanical behaviors. Flow diagnostics offers a natural pre-processing step that complements modern coupled hydromechanical reservoir simulation, uncertainty quantification, and optimization workflows.

Contaminant transport and remediation in subsurface, acidization to enhance permeability in oil recovery, and packed bed reactors in chemical engineering, are the typical applications and phenomena of reactive transport of solutes in porous media. A principal scientific problem in subsurface reactive transport is to determine the effective reaction rates from the pore-scale upwards. In "Scale-dependence of reaction rates in porous media \& physical and chemical heterogeneity", Branko Bijeljic, from Imperial College London, presented a new methodology, termed screening pore-scale imaging and modelling, to predict the fluid/solid reaction rates based on the systematic characterization of both physical and chemical heterogeneity in multi-mineral systems. Based on this method, 3D multispecies fluid/fluid reversible reactive transport in a micro-CT image of carbonate rock is simulated to entail 
spatially resolved information on connected micro-porosity. He concluded that reactive behavior in porous media is simultaneously influenced by pore space heterogeneity, multispecies reactive transport, and reaction reversibility.

\section{Writing workshop: How to write a successful research paper}

In the writing workshop "The 10 mistakes you should avoid", J. Jaime Gómez-Hernández, from the Polytechnic University of Valencia, addressed the younger audience of InterPore2020 on the critical task of how to write a scientific paper. Using a case-based and flipped approach, Jaime presented bad examples of paper submission from his experience as a member of the Editorial Board of Mathematical Geosciences for the last ten years. Specifically, the common ten mistakes discussed are:

- To write in a language that looks like English, but it is not English

- Sloppiness, carelessness

- Choice of an inappropriate journal

- Lack of originality

- Don't get too smart

- Inconclusive conclusions

- (Self) Plagiarism

- Lack of clarity

- Poor bibliography

- Poor state-of-the-art

- Not believing in your own work

The seminar ended with a discussion of what to do when a manuscript requires a major revision or it is rejected but the authors are convinced of the quality and originality of their work.

\section{Career development event}

The Student Affairs Committee organized a Career Development Event during this year's InterPore conference. This event followed the overall structure of similar events that were organized in previous years in the physical conferences. This event has always been very well received, it also received some good attentions and positive feedbacks from the community even with the new challenges of the online format this year. Three researchers were invited, followed very different career paths in academia/industry, to share their experience with the community of students and early career researchers. Tiina Roose, from University of Southampton, Veerle Cnudde, from Ghent University and Utrecht University, and Benoît Noetinger, from IFP Energies Nouvelles, submitted their prerecorded presentations which could be followed anytime by the attendees. A live Q\&A session was also organized, where the attendees could directly interact with the three speakers, asking questions about the challenges of the different career paths and obtaining a direct personalized feedback from them. This is very important, since there are many early career researchers who, in spite of succeeding at their present activity $(\mathrm{PhD}$, postdoc, etc.), struggling with planning the future steps of their careers. There is plenty information available about different challenges in porous media science, but many young researchers find it hard to understand the personal challenges faced by the people running the science. Not everyone is lucky enough to be close to a set of different professionals who are willing to have this important conversation. InterPore's career development event aims precisely at filling that gap and we are very grateful to the speakers of this year's event who did a truly excellent job. It would like to highlight the tremendously important issue of gender imbalance in science, technology, engineering and mathematics (STEM) that was eloquently raised by one of the speakers this year, Tiina Roose. It is easy to understand the issue, but sometimes people find it hard to turn this understanding into active measures to help mitigate the problem. We believe this is an important conversation to have the initial pathway for bringing the topic to InterPore this year. We hope that this conversation won't stop and this initial input can act as a trigger for a more thorough discussion and action to raise awareness and act against the under-representation of women in the STEM fields.

\section{Acknowledgements}

The information presented here is from the link of InterPore2020. The great work of the conference Program and Organization committees is highly acknowledged. Also thanks goes to the participating scholars who actively participated, studied recorded presentations, left comments and questions and attended the live Q\&A and keynote/invited sessions. It was a remarkable and rich academic feast. Dr. Karolin C. Weber from InterPore Executive Office, Prof. J. Jaime GómezHernández from the Polytechnic University of Valencia, Prof. Marcel Moura from University of Oslo are also appreciated for providing important materials of this conference.

\section{Conflict of interest}

The authors declare no competing interest.

Open Access This article, published at Yandy Scientific Press on behalf of the Division of Porous Flow, Hubei Province Society of Rock Mechanics and Engineering, is distributed under the terms and conditions of the Creative Commons Attribution (CC BY-NC-ND) license, which permits unrestricted use, distribution, and reproduction in any medium, provided the original work is properly cited.

\section{References}

Bear, J., Bachmat, Y. Introduction to Modeling of Transport Phenomena in Porous Media. Berlin, Germany, Springer Science \& Business Media, 2012.

Darcy, H. Les Fontaines Publiques de la Ville de Dijon. Paris, France, Dalmont, 1856.

Das, D.B., Hassanizadeh, S.M. Upscaling Multiphase Flow in Porous Media. Berlin, Germany, Springer, 2005.

Ingham, D.B., Pop, I. Transport Phenomena in Porous Media. Amsterdam, Netherlands, Elsevier, 1998.

Vadász, P. Emerging Topics in Heat and Mass Transfer in Porous Media: From Bioengineering and Microelectronics to Nanotechnology. Berlin, Germany, Springer Science \& Business Media, 2008.

Vafai, K. Handbook of Porous Media. Baca Raton, USA, CRC Press, 2015. 